\title{
ADLATUS Interface CD für Schulen am Beispiel der Erdatmosphäre: Ein Pilotprojekt
}

\author{
G. K. Hartmann ${ }^{1}$, A. Nölle ${ }^{2}$, G. Dettmer ${ }^{3}$, and E. Putz ${ }^{4}$ \\ ${ }^{1}$ Max-Planck-Institut für Aeronomie (MPAe), Max-Planck-Str. 2, 37191 Katlenburg-Lindau, Germany \\ ${ }^{2}$ ADLATUS Team-Manager, Science-Softcon, Maintal \\ ${ }^{3}$ Konrad Adenauer Schule (KAS), Seligenstadt (Hess.) \\ ${ }^{4}$ IGAM, Universität Graz, Österreich
}

Zusammenfassung. The goal of the presented pilot project is the creation of a modular interactive online and offline interface on $\mathrm{CD}$ which can be used between schools, various information sources and the science community, i.e. actual, new research results should be made available for students from elementary schools to high schools in an uncomplicated - school and age specific - form. At the beginning this interface will be realised using as an example the existing knowledge about the Earth atmosphere followed by other topics like (drinking) water, energy, soil, and raw material etc. The interactive, interface concept "ADLATUS for schools", which is a further development of the scientific DUST-2 CD, however, complements and interrelates for the first time this information with a so called "local, school specific" part - about $20 \%$ of the CD storage space will be reserved for that - to be generated according to the "individual" school and environmental conditions in special co-operative teaching and learning teams. For the selected topic the ADLATUS CD will contain global and regional background information, examples for time series data, links, and software (freeware and shareware), especially for an interactive graphic representation of the information. The $\mathrm{CD}$ should be updated at least every second year. It will document the results of a direct dynamic interrelationship between the geo-science community, elementary schools and other school types. It will be beneficial for the future of both and other science domains that will follow.

Ziel des hier vorgeschlagenen Pilotprojekts ist die Erstellung einer modular aufgebauten interaktiven online und offline Schnittstelle (Interface) auf $\mathrm{CD}$, die Schulen mit verschiedenen Informationsquellen und der Wissenschaftsgemeinschaft verbinden kann und so aktuelle wissenschaftliche Forschungsergebnisse den Schülern von der Grundschule bis hin zum Gymnasium in möglichst einfacher, verständlicher und altersgemäßer Form vermittelt. Dieses Interface

Correspondence to: G. K. Hartmann

(ghartmann@linmpi.mpg.de) soll in einer ersten Stufe am Beispiel unseres Wissens über die Erdatmosphäre realisiert werden, gefolgt von den Themen (Trink)Wasser, Energie, Boden und Rohstoffe. Das interaktive Interface-Konzept "ADLATUS für Schulen", eine Weiterentwicklung der DUST-2 CD - vorwiegend für die Wissenschaftsgemeinde gedacht - ergänzt und verknüpft zum ersten Mal diese Informationen mit einem "schulspezifischen Lokalteil", für den etwa $20 \%$ des CD Speicherplatzes vorgesehen werden, und der von den einzelnen Schulen lehrplanspezifisch, lehrerspezifisch und ortspezifisch in Lehr- und Lernteams selbst "erzeugt" werden soll. Die ADLATUS CD wird zu dem ausgewählten Thema globale und regionale Hintergrundinformationen, Zeitreihendaten, Links, und Software (Shareware oder Freeware) enthalten, insbesondere zur interaktiven graphischen Darstellung der Informationen. Sie soll spätestens alle zwei Jahre auf den neuesten Stand gebracht werden. Sie wird die Ergebnisse einer direkten, dynamischen Wechselwirkung zwischen der Geo-Wissenschaftsgemeinschaft, den Grundschulen und anderen Schultypen dokumentieren und für die Zukunft beider nützlich sein - ebenso für die anderen nachfolgenden Wissenschafts- und Technikbereiche.

\section{Vorbemerkung}

Dieser Vortrag bei der Kleinheubacher URSI Tagung am 1.10.2002 soll in der Bundesrepublik Deutschland längerfristig dazu beitragen:

1. Den großen Informationswachstumsproblemen so wie der Verringerung der personellen und finanziellen Ressourcen für die Atmosphärenforschung weniger unzureichend entgegenzuwirken. (Im Forschungsbereich Erdatmosphäre ist der zunehmende Mangel an aktueller, qualifizierend gefilterter, verständlicher (sekundär) Information trotz exponentiell anwachsender potentieller (primär) Information (z.B. Rohdaten) mit am stärksten spürbar). 
2. Den negativen Ergebnissen der Pisa-Studie stärker entgegenzuwirken, und zwar durch das Konzept: "ADLATUS Interface CD für Schulen". (ADLATUS: Gehilfe). 1) und 2) sind untrennbar voneinander und wechselwirken miteinander.

\section{Einleitung}

Der "Output" eines Informationssystems kann nicht besser sein als sein "Input". Deshalb ist ein wesentlicher Teil der heutigen Informationsprobleme bedingt durch unzureichende Aufmerksamkeit (und unzureichende Ressourcen) bei der "Erschließung" und Archivierung (Speicherung) der erworbenen oder selbst generierten Daten. Informations-Retrieval Probleme

1. Es wird zuviel Information geliefert, d.h. die Verkleinerung scheitert am Fehlen von weiteren Stichworten.

2. Es wird zu wenig Information geliefert d.h. die Bedeutungsumgebungen werden unzureichend beschrieben.

3. Es wird keine Information geliefert, obwohl sie in dem System gespeichert ist, d.h. die Form der Wissensrepräsentation ist unzureichend ebenso wie die Kontrolle der Bedeutungsveränderungen bei den "Transformationen" (den unvermeidbaren Filterprozessen).

\section{Abhilfe}

1. Die Befragung kompetenter (weiser) Experten oder Expertinnen bzw. entsprechender Bibliothekare oder Bibliothekarinnen, wäre wohl die optimalste Lösung, aber auch eine immer unwahrscheinlicher zu realisierende.

2. Andere Verbesserungsmöglichkeiten:

- Erweiterung der Repräsentation der relevanten Information

- (Automatische) Indexierung mehrwertiger Kontexte

- Weniger unzureichende Bedeutungserhaltung während des Transfers, d.h. dem Filterprozess, von der (Such)-Anfrage bis zur Antwort

- Verbesserung der Informationsausgabe

3. Begriffliches Informations-Retrieval System (BIRS) abgeleitet aus der Formalen Konzeptanalyse (FCA) mit Einbeziehung von Wörterbüchern und Thesauren. Es kann optimal den Ist-Zustand, erfassen und darstellen, besonders unter Verwendung der TOSCANA Software (Kollewe et al., 2000; Wolff et al., 2000).

4. SomAccess - Ein Softwareprototyp zur interaktiven Navigation in Text-Datenbanken; mit Einbeziehung von Wörterbüchern und Thesauren. SomAccess kann optimal die Veränderungen erfassen und darstellen (Nürnberger et al., 2001).
5. Dynamische, kleine Sammlungen konkreter und abstrakter Begriffe im Zusammenhang mit wichtigen konkreten (Alltags)-Begriffen (Hartmann, 1985), z.B. in einem Thesaurus (Erd)-Atmosphäre oder (Trink)Wasser $\left(\mathrm{H}_{2} \mathrm{O}\right)$ oder Boden oder Energie, oder Rohstoffe usw. (Für Atmosphäre und Trinkwasser gibt es zwei Entwürfe). Dadurch wird eine bessere Verknüpfung der komplementären Methoden 3. und 4. möglich, aber auch eine unkompliziertere Klassifizierung bzw. eine bessere multikontextuale Indexierung eigener und fremder Informationen (Daten).

3., 4., und 5. sind sehr wichtige, wenngleich unauffällige Elemente der DUST-2 CD und des weiterführenden ADLATUS Konzeptes und damit auch des Pilotprojektes "ADLATUS für Schulen".

Die Suche nach einer allgemeinen (universalen) Ordnung der Information ist bisher erfolglos geblieben. Die Begegnung mit anderen nicht europäisch geprägten Kulturen zeigt, dass nicht alle Menschen, d.h. weltweit, gewiss sind, daß der Sinn so zu Wort kommen kann, dass wir annehmen dürfen, er sei allgemein, und das heißt doch für alle gemeinsam gültig. Das von Niels Bohr (1928) wieder entdeckte Komplementaritätsprinzip - es gilt nicht nur in der Physik, sondern auch in anderen Bereichen, z.B. der Sprachphilosophie - ist der Hauptgrund dafür, dass sie wahrscheinlich auch in der $\mathrm{Zu}$ kunft erfolglos bleiben wird. Um die negativen Folgen des exponentiellen Informationswachstums, d.h. des velociferischen* Trends, verringern zu können, wird es in der Zukunft deshalb besonders darauf ankommen, neue interaktive (online und offline) "Interface-Module", z.B. "ADLATUS CD ROMs" zu schaffen: 1) zum effektiveren Umgang mit eigener oder fremder Information, 2) zum unkomplizierteren, effektiveren, nutzerfreundlicheren online Zugang zu (wichtigen) schon vorhandenen Dokumentations- und Informationssystemen, 3) zu mehr offline Nutzungsmöglichkeiten dieser (heruntergeladenen) Information, z.B. "zu Hause". Dabei muss sowohl das Gemeinsame als auch das Unterschiedliche innerhalb und zwischen den Disziplinen, Kulturen und Generationen erfasst und dargestellt werden können, vor allen Dingen aber die wichtigen offenen Fragen. Sonst riskieren wir als Folge des großen Informationswachstums zunehmend mehr Informationsverweigerung und "Deformation" statt eines Zugewinns an mehr aktueller (verständlicher und schnell nutzbarer) Information.

*Der Begriff velociferisch wurde von J. W. von Goethe 1825 aus den Begriffen Velocitas (Geschwindigkeit) und Luzifer (Teufel) zusammengesetzt, um die Gefahren der sich beschleunigenden Veränderungen zusammenfassend beschreiben zu können.

Bei der Nutzung eines Informationssystems hängt der Informationszugewinn wesentlich davon $\mathrm{ab}$, wie viel man schon vorher wusste. Ohne Vorwissen bzw. a priori Annahmen und mit starrem Vorurteil gibt es keinen Zugewinn an Information, man ist nachher nicht "schlauer" als vorher (Beck-Bornholdt und Dubben, 2001). Ähnliches wissen wir auch schon aus der Filtertheorie. Das Pilotprojekt 
"ADLATUS für Schulen" basiert deshalb auf der Nutzung und dem Aufbau von offenen, lernfähigen Informationssystemen, der Synergie von Mensch und Computer und interdisziplinärer Teamarbeit. Das Pilotprojekt soll mit dem Bereich Erdatmosphäre beginnen, weil dort die beteiligten drei Wissenschaftler über langjährige Erfahrungen verfügen und weil dort das Informationswachstum mit am größten ist, zumindest nach dem Start der Erdbeobachtungssatelliten der neuen Generation. Dazu gehört "ENVISAT", der am 1. März 2002 erfolgreich gestartet wurde. Gleichzeitig sollen aber auch Vorarbeiten für weitere konkrete (alltagsund lehrplanbezogene) Themenbereiche erfolgen wie: (Trink)-Wasser, Boden, Energie und Rohstoffe. Durch die so erreichte größere Betroffenheit der Grundschüler wird längerfristig auch die Atmosphärenforschung wieder mehr Anerkennung und Unterstützung finden. Damit wird sich der gegenwärtige Abwärtstrend langsam wieder umkehren. Er ist nicht nur durch die schlechte wirtschaftliche Lage bedingt, sondern er ist auch eine Folge des unzureichend berücksichtigten demographischen Zyklus und der Tatsache, dass die Verfügbarkeit von zuverlässigen "Atmosphärendaten" im Laufe der letzten Jahrzehnte zu selbstverständlich geworden ist.

\section{Das Informationswachstumsproblem am Beispiel der Erdatmosphäre und einer Internetsuche.}

Am Beispiel der Atmosphärenforschung kann man den Überfluss an "potentieller" Information (Rohdaten, primär Information) und die (exponentielle) Wachstumsrate deutlich machen. Auf diesem Gebiet gab es schon 1990 mehr als $2,5 \times 10^{14}$ Bit an Information, mit einer jährlichen Wachstumsrate von etwa $10 \%$. Bei einer Seitenstärke von $0.1 \mathrm{~mm}$ ergibt das eine (fiktive) Buchreihe mit dicht bedruckten DIN A4 Seiten von $362 \mathrm{~km}$ Länge. Die neue Generation von Forschungssatelliten wie z.B. ENVISAT - der am 1. März 2002 gestartet wurde - ist so konstruiert, dass sie einen $10 \mathrm{mal}$ größeren Datenfluss erzeugen (können) als die Satelliten Ende der 80er Jahre. Das heißt, dass pro Jahr jetzt ungefähr soviel neue Daten hinzukommen werden wie bis Anfang der 90er Jahre insgesamt gespeichert wurden, das heißt spätestens Ende 2002 hat die Buchreihe eine Länge von etwa $800 \mathrm{~km}$ erreicht. Das entspricht ungefähr der Entfernung von Hamburg nach München. Das wird unvermeidbar zu neuen, nur unvollkommen standardisierbaren Datenbasen führen, deren effiziente Nutzung von den oben erwähnten, gleichzeitig zu schaffenden Schnittstellen abhängen wird, wie z.B. von dynamischen, interaktiven DUST-2 oder ADLATUS CD ROMs. Der Mangel an aktueller, verständlicher, qualifizierend gefilterter (sekundär) Information wird schleichend immer größer, weshalb es auch so schwer ist, dem wirkungsvoll zu begegnen. (Die großen Fortschritte der Mikroelektronik und der Digitaltechnik tragen so auch zu dem wachsenden "Digital Divide" bei, besonders stark in den nicht hoch industrialisierten Nationalstaaten). Ein anderes Beispiel von einer sehr großen Informationsmenge zeigt die folgende INTERNET Suche mit einer konventionellen Suchmaschine (LY-
Tabelle 1.

\begin{tabular}{cc}
\hline Suchfrage & Treffer \\
\hline Water & 1858896 \\
Water vapour & 4707 \\
Water vapor & 35206 \\
$\mathrm{H}_{2} \mathrm{O}$ & 23301 \\
Atmosphere & 290324 \\
Water and Troposphere & 1695 \\
Stratosphere und Water & 2652 \\
Water and Atmosphere & 65189 \\
\hline
\end{tabular}

COS) im Oktober 1999.

Es gibt aber auch bei fachspezifischen Informationssystemen Probleme (Weber et al., 2000). Man schätzt, daß die Menschheit gegenwärtig etwa $10^{18}$ Bit an Information jährlich erzeugt. Die physikalische - thermodynamisch bedingte - Grenze für die von Menschen erzeugbare Menge von Informations-Bit im System Sonne-Erde-Weltraum liegt noch 25 Größenordnungen ( $10^{43}$ Bit pro Jahr) darüber, ist also noch sehr weit entfernt. Dies gilt allerdings nicht für die biologischen Grenzen der Fähigkeiten des Menschen, Information zu speichern und zu verarbeiten. Sie sind schon sehr nahe gerückt und verursachen jetzt schon große Probleme. Die rasche Entwicklung der Rechnersysteme begünstigte die drastische Vermehrung der angebotenen Informationsmengen, da der elektronischen Aufbereitung und Speicherung wenig Widerstände entgegenstanden. Inzwischen ist aber klar geworden, dass die Bewältigung dieser Informationsflut ganz neuartige Anforderungen an die Nutzer - allerdings auch an die "Erzeuger" - solcher Daten stellt, nicht zu vergessen auch an diejenigen Institutionen, die solche Daten - bibliographische und/oder numerische speichern und (nutzerfreundlich) zur Verfügung stellen (sollen) wie die nationalen und internationalen Datenzentren.

\section{ADLATUS für Schulen: Text des Flyers}

Flyer Text ADLATUS für Schulen: Ein Pilot-Projekt in Zusammenarbeit von: Konrad-Adenauer-Schule (KAS)*; Max-Planck-Institut für Aeronomie**; sience-softCon*** Durch das Pilot-Projekt soll Schülern die Möglichkeit gegeben werden, unter pädagogischer Anleitung intensiveren Kontakt mit Naturwissenschaft, Technik und neuen Medien zu bekommen.

\section{Probleme}

Es gibt sehr viel und schnell zunehmende regionale und globale naturwissenschaftlich-technische Information. Sie macht die meisten Schüler kaum betroffen. Lokale, aktuelle konkret betroffen machende Information ist unzureichend verfügbar und bisher auch wenig in Schulen nutzbar. Diese Information ist aber unverzichtbar, um mehr lokales Wissen und den Umgang damit (lokale Kompetenz) aufzubauen, 
die notwendig ist, um nicht nur die regionalen und globalen Informationen besser $\mathrm{zu}$ verstehen, sondern auch um aus der Informationsflut das für den eigenen Bedarf Wichtige besser und schneller herausfiltern und praktisch umsetzen $\mathrm{zu}$ können. Aus ökonomischen Gründen ist Abhilfe von oben, hier z.B. von den großen nationalen und internationalen Datenzentren und Verlagen kaum zu erwarten. Es ist also eine lokale Aufgabe.

\section{Ziele}

Mehr lokale Kompetenz bei den Schülern schaffen durch interdisziplinäre Zusammenarbeit eines engagierten, kompetenten vorwiegend lokalen Teams unter Einsatz moderner Kommunikationsmedien. Verstärktes praktisches Erleben in ernsthaftem Spiel oder spielerischem Forschen und Entdecken - der Schüler im naturwissenschaftlich-technischen Unterricht.

\section{Wege}

Die neuen Medien, z.B. die PCs, CDs, Internet usw. bieten wichtige, ergänzende Hilfsmittel für den Schulunterricht. Sie werden durch das ADLATUS Konzept besonders effizient genutzt. Ein gut zusammenarbeitendes interdisziplinäres, motiviertes und kompetentes Team bestehend aus Lehrern, Schulleitung, Elternvertretern sowie Wissenschaftlern und Ingenieuren ist die Hauptvoraussetzung für ein erfolgreiches Umsetzen des ADLATUS-Konzeptes in der Grundschule. Das ist bei dem KAS-ADLATUS-Team unerwartet gut gegeben.

\section{Warum ein ADLATUS Pilotprojekt bei KAS?}

Weil dort ein besonders gutes (synergistisches), interdisziplinäres, engagiertes Team dafür zur Verfügung steht, das im Bereich Naturwissenschaft und Technik "Mehr kreatives, kooperatives Lehren und Lernen im Team" und mehr lokale Kompetenz ermöglichen wird. Weil personelle, materielle und finanzielle Voraussetzungen für eine erste Phase des Projektes vorhanden sind, und zwar aus einer überschaubaren Region mit gewerblichen und industriellen Betrieben.

\section{Ausblick}

Besonders wichtig an ADLATUS ist das lebendige (dynamische) Konzept, konkretisiert durch das synergistische KAS-ADLATUS Team. Sehr wichtig sind ferner die ADLATUS CDs zusammen mit den "Multi Media Werkzeugen" in der Schule. Die CDs sollen eine leicht handhabbare interaktive Schnittstelle realisieren, besonders um einen nahezu stufenlosen Übergang des ADLATUS- Konzeptes von der Grundschule zu anderen Schultypen zu ermöglichen. Die CDs sollen spätestens alle zwei Jahre aktualisiert und kostengünstig öffentlich verfügbar gemacht werden. Die Finanzierung dieses Pilot-Projektes erfolgt vorwiegend durch private Spenden und/oder Werbebeiträge.

\section{Ehrenamtliche Beiträge}

Wissenschaftlich-technische Beratung durch Gerd K. Hartmann (Dr., Prof.), MPAe

Vertreter der Elternschaft der KAS

Umweltamt der Stadt Seligenstadt

\section{Kontakte und weitere Infos:}

- Konrad-Adenauer-Schule (KAS) - Gudrun Dettmer, Rektorin, Steinweg 21, 63500 Seligenstadt, kasseligenstadt@t-online.de,www.kas-seligenstadt.de

- Max-Planck-Institut für Aeronomie (MPAe) - Dr. Gerd K. Hartmann, Max Planck Str. 2, 37191 Katlenburg-Lindau, ghartmann@linmpi.mpg.de, www.linmpi.mpg.de/english/projekte/mas/dust-2

- science-softCon - Dr. Andreas Nölle, Auf der Burg 4, 63477 Maintal, andreas.noelle@science-softcon.de, www.science-softcon.de

\section{Von DUST-2 zu ADLATUS für Schulen}

Das DUST-2 Konzept wird in einem weiteren Schritt zum ADLATUS (Helfer, Assistent) - Konzept erweitert, in einem ersten Schritt zu einem "ADLATUS für Schulen" der beginnend mit den Grundschulen schrittweise auch alle anderen Schultypen umfassen soll bis hin zur Wissenschaft. Folgende Schritte sind geplant:

A Lokalteil des ADLATUS für Schulen (in unserer Muttersprache Deutsch), der ungefähr 1/3 des Speicherplatzes der CD ROM ausmachen sollte, bei dem die Hauptarbeit in und von der (Grund)-Schule - koordiniert von der Schulleiterin z.B. von Gudrun Dettmer (KAS) - gemacht werden muss. Hier gibt es nur unzureichende Vorarbeiten, deshalb muss mit diesem Teil A zuerst begonnen werden, und zwar:

1. Mit der "Abbildung" der Themen auf den Lehrplan der Grundschule, unter Berücksichtigung der Kompetenz und des Engagements der Lehrer sowie der lokalen Umweltbedingungen und schulspezifische Schaffung und Wartung der IT-Infrastruktur.

2. Damit dass die Schule nicht nur externe Daten nutzt, sondern in Teamarbeit im Unterricht auch eigene "erzeugt" und davon ausgewählte im Lokalteil der CD speichert. Damit erhöht sich wesentlich die "lokale" Kompetenz.

3. Mit dem Fundraising - es wurde vor kurzem für die KAS (Seligenstadt; Hess.) begonnen - und Durchführung der ersten Phase des Pilotprojektes. (Die zweite Phase beinhaltet auch die Verknüpfung mit dem Globalteil und dessen Weiterentwicklung). 
Das interdisziplinäre ADLATUS-Team dieses KAS Pilotprojektes beabsichtigt, bei der nächsten Kleinheubacher Tagung im Jahre 2003 über die Fortschritte berichten.

B Globalteil des ADLATUS für Schulen, der etwa 2/3 des Speicherplatzes ausmachen sollte und für den einige wichtige Elemente und Ideen von der DUST-2 übernommen werden sollen, und zwar:

1. Ergänzende, interaktive, nutzerfreundliche Beispiele von Daten, ihrer qualifizierenden Filterung und ihren Validationsprozessen sowie der wichtigsten zugrunde liegende wissenschaftlich-technischen Prozesse mit entsprechender Dokumentation - einschließlich Thesaurusaufbau und Thesauruspflege.

2. Zweisprachigkeit, weil Englisch in Deutschland nicht die Muttersprache ist, in dem betroffenen Themenbereich Naturwissenschaft und Technik heute aber Englisch die "Hauptsprache" ist. Das gilt in diesem Fall für alle Texte sowie den entsprechend ergänzten Glossar, die ADLATUS-Dokumentation und für das Literaturverzeichnis.

3. Verknüpfung der Daten ("Antworten”) mit den vorausgehenden Fragen vor ihrem kulturellen Hintergrund. (Nur wer die Fragen versteht, versteht auch die gegebenen Antworten so, dass er sinnvolle, neue Fragen stellen kann). Hier liegt eine besondere wissenschaftlich, didaktische und pädagogische Herausforderung.

4. Regelmäßige zuverlässige Pflege der ADLATUS Version, Aktualisierung der Daten und der Dokumentation sowie aufwärtskompatible Anpassung des Konzeptes an die (schnellen) Veränderungen der IT-Technologie. Das gilt sowohl für die Verwendung von ADLATUS in der Wissenschaft als auch in der Bildung.

Ständige Erfolgskontrollen auf verschiedenen Ebenen, z.B. durch Auswertung von Nutzerprofilen, Nutzerhinweisen, Nutzerfragen usw. Die Interface- bzw. Filter-Funktion der verschiedenen ADLATUS Versionen werden in dem "Globalteil" auch das Ungleichgewicht zwischen populistischer und sehr spezieller wissenschaftlicher Literatur verringern.

\section{SomAccess, FCA und TOSCANA auf der DUST-2 CD}

Das exponentielle Wachstum der "potentiellen" Information (z.B. Rohdaten) und der wachsende Mangel an "aktueller" (qualifizierend gefilterter, schnell nutzbarer) Information, das in den letzten Jahrzehnten in den Industriestaaten zu beobachten ist, macht ergänzende Methoden des Informationsmanagements immer unverzichtbarer, insbesondere in den Bereichen: Klassifikation, qualifizierendes Filtern (auswählen), Wiederauffinden (Retrieval) und Informationsdarstellung, z.B. graphische Darstellung der Ergebnisse. Es wird immer deutlicher, dass die Suche in einem Informationssystem keine besseren Ergebnisse liefern kann als der sog. "Input" in das System, das kann letztendlich durch kein noch so kompliziertes Retrievalverfahren umgangen werden. So sollte ADLATUS für Schulen schon in den Grundschulen helfen, sowohl den Umgang mit der Datenarchivierung (Klassifizierung) als auch mit der Datenwiederauffindung (Datenretrieval) zu lernen. Gegenwärtig besteht ein großes Ungleichgewicht zwischen Archivierung/Klassifizierung und Retrieval zugunsten des letzteren. Auf der DUST-2 CD sind neben der DUST-2 Software folgende (ergänzende) Methoden dargestellt.

A Das SomAccess ${ }^{1}$ Verfahren (Klose et al., 2000; Hartmann et al., 2000a; Nürnberger et al., 2002) wurde angewandt auf:

1. Ergebnisse einer Stichwortsuche nach « Ozon / ozone $\gg$ an die INSPEC-Datenbank. Aus dieser Abfrage standen die Titel, Abstracts und Keywords von 3165 Dokumenten zur Verfügung.

2. Abstracts der Konferenz EGS 2000. Der mit SomAccess vorgestellte Ansatz kann eine sehr sinnvolle Ergänzungen klassischer Suchverfahren sein, insbesondere bei Datenbasen oder Problemstellungen, bei denen ein Nutzer darauf angewiesen ist, effizient auch nach verwandten Dokumenten suchen zu können. (Suche in der "Dokumentenumgebung") und die Ergebnisse nutzerfreundlich, z.B. graphisch, darstellen möchte. Interessant sind in diesem Zusammenhang die Beziehungen zwischen der bei SomAccess verwendeten Wortkategorienkarte - einer graphischen Darstellung der "Ergebnisse" - und "Thesauren". So ist es denkbar, die Definitionen von Synonymen und Äquivalenzklassen eines Thesaurus des jeweiligen Wissensgebiets zur Verbesserung der Wortkategorienkarte zu verwenden. Auf der anderen Seite kann das automatische Lernen der Wortkategorienkarte den manuellen Aufbau und die Aktualisierung bestehender Thesauri unterstützen. Durch Ausnutzung von Synergieeffekten könnte sowohl die assoziative Informationssuche als auch die meist aufwendige und kostspielige Dynamisierung - im angelsächsischen auch "upgrading" genannt - von (kulturspezifischen) Thesauren verbessert werden. Die wachsenden Informationsprobleme machen immer deutlicher wenn auch nur langsam - wie notwendig, aber unzureichend bisher neue Thesaurusentwicklungen bzw. Verbesserungen alter Versionen durchgeführt werden (können). $\mathrm{Zu}$ einer wesentlichen Verbesserung kann hier auch TOSCANA (Kollewe et al., 2000) beitragen,

\footnotetext{
${ }^{1}$ SomAccess gehört zur Gruppe der sog. "bottom-up” Informationsmanagementmethoden, FCA zur komplementären Gruppe der "top-bottom" Methoden - zu der auch die "Ontology" gehört, die im Rahmen der künstlichen Intelligenz entwickelt wurde. Siehe dazu auch: http://www-ksl.stanford.edu/kst/what-is-an-ontology.html (Thomas R. Gruber, 1994) und

(John F. Sowa, 1997) http://www.cs.utexas.edu/users/mfkb/related. html.
} 
das auch zur graphischen Darstellung der FCA Ergebnisse verwendet wird.

B Das Verfahren der Formalen Konzeptanalyse - FCA: Formal Concept Analysis - (Wolff et al., 2000; Hartmann et al., 2000a) wurde angewandt auf gemessene (MAS)-Ozondaten. Seine sehr guten, bisher kaum erreichten, selektiven "Filtereigenschaften" konnten gezeigt werden. FCA kann und soll allerdings auch für Textretrieval eingesetzt werden.

SomAccess, FCA und ein fachspezifischer Thesaurus sollten deshalb synergistisch kombiniert werden. Dies hat die Weiterführung der DUST-2 Aktivitäten in den letzten Monaten gezeigt. Da die am "ADLATUS für Schulen" beteiligten Wissenschaftler über langjährige Erfahrungen auf dem Gebiet der Atmosphärenphysik verfügen, wurde die Erdatmosphäre als erstes Thema ausgewählt, das durch weitere ergänzt werden soll und muss, z.B. Trinkwasser, Energie, Boden, Rohstoffe, Biosphäre usw. Es wurde für den Bereich Erdatmosphäre ein "Basisthesaurus" entworfen, der sowohl eine Nutzung vom Konkreten zum Abstrakten als vom Abstrakten zum Konkreten ermöglicht und der mit den oben erwähnten Methoden (kulturspezifisch oder schulspezifisch) weiterentwickelt werden kann. In dem Masse wie das Informationsangebot in diesem Bereich zugenommen hat geht es immer mehr sowohl um das kompetente Erfassen, Archivieren und Wiederauffinden schulspezifischer, lokaler Informationen (numerische Daten und Texte) als auch ausgesuchter externer Informationen. Am stärksten herausgefordert wird wohl die Atmosphärenforschung, auf der Datenseite z.B. durch den Start von Satelliten der neuen Generation. ENVISAT wurde am 1. März 2002 erfolgreich gestartet. Aber auch textseitig wächst die Herausforderung durch "e-publishing mit online Zugang" (Hartmann, 2002) sowie durch neue interaktive Informationssysteme. Eine besondere Rolle wird dabei ESPERE spielen - http://www.espere.net. Das ADLATUS-Team strebt noch in diesem Jahr eine engere Zusammenarbeit mit dem ESPERE-Team an. Die dynamische Interface-Funktion des "ADLATUS für Schulen" soll möglichst unkompliziert und selbstkonsistent beides ("Lokalteil und Globalteil") zuverlässig unterstützen. Deshalb sollte jetzt beides begonnen werden und iterativ weiterentwickelt werden, gegenwärtig der "Lokalteil" allerdings vorrangig. Dazu bedarf es allerdings noch interdisziplinärer Entwicklungsarbeiten zwischen der Schulleitung, den Lehrern und den beteiligten Wissenschaftlern, die nach den Erfahrungen der letzten Jahre kaum Aussicht auf Finanzierung von "oben", d.h. von staatlichen Institutionen haben. Deshalb soll ein nicht-staatliches "Fundraising", insbesondere von der Schulelternschaft von "unten" - begonnen werden, so dass ADALTUS für Schulen auch eine schulspezifische Einrichtung, Einweisung und Wartung der IT-Infrastruktur umfassen kann. Die Probleme des Wachstums der Information sind stetig mit den Fortschritten der IT-Technologie gewachsen, werden aber bisher wie alle schleichend zunehmenden Probleme nur unzureichend "bekämpft". Wenn man nicht erst auf eine Katastrophe warten will, die häufig zu viel unreflektiertem Aktionismus führt, dann muss man dem Problem frühzeitig und geduldig (antivelociferisch) in kleinen (meist unspektakulären) Schritten entgegenwirken und dort beginnen, wo die "Behandlung des Themas" weniger durch starre Vorurteile behindert werden kann oder deshalb unberücksichtigt bleibt, da es sich ja um "Selbstverständliches" handelt. Grundschulen sind deshalb besonders dafür geeignet, bisher allerdings in naturwissenschaftlich-technischen Belangen nur unzureichend einbezogen worden. Selbst wenn man so Katastrophen nicht verhindern kann, dann wird im "SchulTeam" durch offenen Umgang mit der "Gefahr" wenigstens Angst abgebaut und es werden die "Überlebenschancen" der meisten Teammitarbeiter erheblich verbessert - Arche Noah-Effekt. Selbstverständlich scheint - zumindest für die meisten Erwachsenen über 20 - zu sein, dass wir immer mehr und "bessere" Informationen über die Erdatmosphäre und das solar-terrestrische System bekommen und immer auf beratende (kompetente) Experten zurückgreifen können und "lokale, individuelle Kompetenz" kaum benötigt wird. Deshalb werden seit einigen Jahren in der Bundesrepublik Deutschland, allerdings auch anderswo, und abgesehen von einigen Risikoversicherungen - die Investitionen für Grundlagenforschung im Bereich Atmosphärenforschung, einem Teilbereich der Geophysik, stark eingeschränkt. Die Reaktions-Zeitkonstante der Wissenschaftspolitik in den Nationalstaaten ist so groß, dass das Problem erst in einigen Jahren voll sichtbar werden wird, wenn die meisten der heutigen Experten - meist altersbedingt - nicht mehr zur Verfügung stehen werden. Diejenigen, die die Kontinuität und das Know-How bewahren oder überliefern können, gehören offiziell nicht mehr zur aktiven Wissenschaftsgemeinschaft - zumindest in Deutschland. Sie brauchen diese Kontinuität nicht mehr für ihre Arbeit. Gerade diese Generation könnte und sollte - zusammen mit den noch "Aktiven" der vorigen Generation - z.B. im Rahmen von "ADLATUS für Schulen" zur Bewahrung des Wissens über die Erdatmosphäre beitragen. Ähnliches gilt natürlich auch für andere naturwissenschaftlich-technische Gebiete. Es könnten drei Generationen davon Nutzen haben, wenn es dabei wie hier vorgeschlagen auch zu einer modifizierten, verständlichen Weitergabe des Wissens an interessierte, unvoreingenommene und betroffen gemachte Grundschüler kommt. Der geduldige Aufbau lokaler Kompetenz und das "bewahrende Nutzen" globaler würde das Tal, durch das die Atmosphärenwissenschaften gegenwärtig gehen, nicht zu tief werden lassen. Die heutigen Grundschüler in Deutschland könnten zumindest in diesem Bereich (international) nicht nur wieder konkurrenzfähiger werden als es die Pisa-Studie für die vorausgehende Generation zeigt, sondern auch weniger anfällig für des "Kaisers neue Kleider" und das dort erwähnte Pagensyndrom* sowie für mögliche Datenbetrügereien (Hartmann, 2000). So schließt sich der Kreis von der Atmosphärenwissenschaft zu "ADLATUS für Schulen am Beispiel Erdatmosphäre" zurück zur Atmosphärenwissenschaft und rechtfertigt damit wahrscheinlich 
diesen "unwissenschaftlichen" Vortrag, vielleicht unter dem Motto « Die wirkungsvollste Lobby für die Atmosphärenwissenschaft - aber auch andere wissenschaftlich-technische Bereiche - sind unsere Kinder, wenn wir sie rechtzeitig und altersgemäß davon betroffen machen, insbesondere von dem, was wir dabei für selbstverständlich halten $\gg$

*Bemerkung zum Begriff Pagensyndrom:

Der Begriff Pagensyndrom wurde von G. K. Hartmann gewählt, zur Kurzbezeichnung des Verhaltens "Aus Angst oder Bequemlichkeit trotz besserer Einsicht am Gewohnten festzuhalten", ähnlich dem Verhalten der Pagen in dem Märchen "Des Kaisers neue Kleider" von Hans Christian Andersen (1898). Nach der Erkenntnis, dass der Kaiser nackt war, schleppten sie die nicht vorhandene Schleppe nur noch um so stolzer hinter ihm her.

Anerkennung. Die Autoren danken ihren Instituten, Firmen und der zuständigen Schulbehörde für die Unterstützung dieser Arbeiten.

\section{Literatur}

Beck-Bornholdt, H. P. and Dubben, H.: Der Schein der Weisen, Hoffmann und Campe, 2. Aufl., 2001.

Hartmann, G. K.: Das Informationssystem OCIR/VIGRODOS, ICC/IIR TAR-IS-OVI-86-01, 1985.

Hartmann, G. K.: Scientific data between validation imperatives, oblivion and fraud, Phys. Chem. Earth (A), 25, 8, 613-618, 2000.

Hartmann, G. K.: Auf der Suche nach einer allgemeinen Ordnung der Information, MPAe-L-853-01-06, 2001.

Hartmann, G. K.: Bridging the digital divide by digital scientific publishing and the ADALATUS CD concept, Invited presentation at the Scientific conference in Taiz, Yemen, October 10th to 13th, 2002, to be published in the proceedings of the Yemeni Science and Research Foundation (YSRF), 2002.

Hartmann, G. K., Nölle, A., Richards, M. L., and Leitinger, R.: DUST-2 CD, Improved Access to Information about the Earth Atmosphere, ISBN 3-936586-02-0, 2000a.
Hartmann, G. K., Nölle, A., and Pfister, G.: Bildungsaspekte: Vom DUST-2 Konzept zum ADLATUS Konzept, Text auf der DUST2 CD: Dokumentation/Bildungsaspekte, 2000b.

Hartmann, G. K., Nölle, A., Richards, M. L., and Leitinger, R.: DUST-2 Towards a more efficient (interactive) documentation and validation of scientific information demonstrated with ozone, water vapour and other selected data of the Earth's atmosphere, Phys. Chem. Earth (A), 25, 8, 607-612, 2000c.

Hartmann, G. K., Kirchengast, G., v. Engeln, A., Richards, M. L., Ramsauer, J., and Jarchow, Ch.: MAS-GRAS sensor combination and optimal estimation retrieval of temperature and $\mathrm{H}_{2} \mathrm{O}$ profiles, Phys. Chem. Earth (A), 25, 8, 625-628, 2000.

Klose, A., Nürnberger, A., Kruse, R., Hartmann, G. K., and Richards, M. L.: Interactive text retrieval based on document similarities. Phys. Chem. Earth (A), 25, 8, 649-654, 2000.

Kollewe, W., Skorsky, M., Hartmann, G. K., Nölle, A., and Richards, M. L.: TOSCANA applied to Thesaurus research, Phys Chem. Earth (A), 25, 8, 643-648, 2000.

Nölle, A., Pfister, G., Seckmeyer, G., Wilhelms, H., Richards, M. L., and Hartmann, G. K.: DUST: An interactive data visualisation tool for selected atmospheric data. Phys. Chem. Earth (A), 25, 8, 635-638, 2000.

Nürnberger, A., Klose, A., Kruse, R., Hartmann, G. K., and Richards, M. L.: SomAccess - Ein Softwareprototyp zur interaktiven Navigation in Textdatenbanken, Künstliche Intelligenz 3/02, pp. 59-64, 2002. (Siehe auch: http://www. kuenstliche-intelligenz.de/)

Robertson, S. and Soboroff, I.: The TREC 2001 Filtering Track Report, available as pdf-file at: http://trec.nist.gov/pubs/trec10/ t10 $\backslash$ underline $\{\sim$ \}proceedings.html Gey, F. C., Oard, D. W., The TREC-2001 Cross-Language Information Retrieval Track: Searching Arabic using English, French or Arabic Queries, available as pdf-file at: http://trec.nist.gov/pubs/trec10/t10 underline $\{\sim\}$ proceedings.html

Weber, K.-H., Hartmann, G. K., and Oberländer, H.: Literature Search on Atmospheric Ozone and Water Vapour from three different Online-Databases, Phys. Chem. Earth(A), 25, 8, 639-641, 2000.

Wolff, K. E., Hartmann, G. K., Nölle, A., and Richards, M. L.: Conceptual knowledge processing and graphical representation of multidimensional atmospheric ozone data, Phys. Chem. Earth (A), 25, 8, 619-624, 2000. 